\title{
ISSUES RELATED TO SPORTS GAMBLING
}

\author{
Robin Insley, Lucia Mok and Tim Swartz *
}

\begin{abstract}
Summary
This paper looks at various issues that are of interest to the sports gambler. First, an expression is obtained for the distribution of the final bankroll using fixed wagers with a specified initial bankroll. Second, fixed percentage wagers are considered where the Kelly method is extended to the case of simultaneous bets placed at various odds; a computational algorithm is presented to obtain the Kelly fractions. Finally, we consider the problem of determining whether a gambling system is profitable based on the historical results of bets placed at various odds.
\end{abstract}

Key words: gambler's ruin, Kelly method, optimal wagering, comparative inference, Gibbs sampling, meta-analysis.

\section{Introduction}

Despite its illegality in many jurisdictions, gambling on the outcome of sporting events is a common activity. For example, Crist (1998) states that Americans illegally wager

${ }^{*}$ Robin Insley is Senior Lecturer, Lucia Mok is an MSc graduate and Tim Swartz is Professor, Department of Statistics and Actuarial Science, Simon Fraser University, Burnaby BC, Canada V5A1S6. Swartz's work was partially supported by a grant from the Natural Sciences and Engineering Research Council of Canada. The authors thank an Associate Editor and two referees whose comments lead to an improvement in the manuscript. 
over $\$ 100$ billion annually on professional and college sports. On the 1999 Superbowl (the championship game of the National Football League), approximately $\$ 87$ million was wagered legally in Las Vegas Sportsbooks (Ordine, 2000). In Australia, commercial gambling on racing alone (horses and greyhounds) resulted in individual losses of $\$ 1.6$ billion in 1997-1998 (Productivity Commission, 1999).

Sports gambling has been increasing over the years (Productivity Commission, 1999) and it is likely that the trend will continue as more government bodies are eager to share in the huge profits that have and can be made. One particular avenue for growth is sports gambling via the internet (Haywood, 2000). Whereas such internet sites are illegal in the United States and in Canada, they are legal and operational in various Caribbean and Latin American countries and in Australia. Internet gambling yields various murky legal issues such as the determination of where the wager is actually placed (e.g. in one's home in North America where the activity is illegal or in the offshore country).

In this paper, we put ethics and legal issues aside and take a look at various practical problems associated with sports betting. We are primarily concerned with issues involving the performance of wagering systems. To a lesser extent, the results are also applicable to financial investments corresponding to an investor who engages in numerous transactions. Many fundamental probabilistic results concerning optimal systems for favourable games are reviewed in Thorp (1969). We are also interested in the problem of identifying profitable gambling systems based on past data.

In section 2 , we provide an introduction to pointspread wagering where we emphasize that it is theoretically possible to develop winning systems. Gambling terminology is explained and the main objective of a bookmaker is discussed. In section 3, we obtain distributional results for the gambler's current bankroll after placing a finite number of bets. This is done in the context of both 'large' and 'small' initial bankrolls. Although the 
results involve only binomial calculations and a simple extension of the classical drunkard's walk problem, they are practical and we have not seen them recorded in the sports gambling literature. Moreover, much of the work concerning the gambler's ruin and related problems was developed long ago with an emphasis on challenging mathematics and approximate solutions. Our approach is based on the realization that today many people have a computer on their desk. In section 4, we turn to fixed percentage wagering where each bet is a fixed percentage of the current bankroll. We provide some theory and a numerical algorithm to obtain optimal fixed percentages in the context of simultaneous bets placed at various odds. The results in this section are extensions of the Kelly system (Kelly, 1956). We also give some distributional results concerning the final bankroll under fixed percentage wagering. In section 5 , we consider the historical results of bets placed at various odds for a proposed gambling system. The practical question arises as to whether the historical data provides evidence that the gambling system is profitable. Profitability entails more than simply checking whether a profit is made in a given year; this could occur by chance. We are instead interested in whether there is long-term profitability in a system. The problem raises inferential issues for which different approaches yield conflicting results. The major inferential problem that arises is the testing of a hypothesis $\Omega_{0}$ versus a hypothesis $\Omega_{1}$ where both $\Omega_{0}$ and $\Omega_{1}$ are 'small' sets relative to the entire parameter space. We propose a non-standard Bayesian approach to the problem which

calculates 'distances' from $\Omega_{0}$ and $\Omega_{1}$ as measures of evidence in favour of each hypothesis. In section 6, we provide a concluding discussion with advice applicable to typical Sportsbook scenarios where upper limits on wagering are imposed.

\section{A primer on betting the pointspread}

There are many types of wagers that can be placed on sporting events (McCune, 1989). Perhaps the most common, is a wager placed against the pointspread. For ex- 
ample, consider a contest between a strong team (Team A) and a weak team (Team B). Whereas popular sentiment may overwhelmingly favour Team A to win, there is typically no concensus on the magnitude of the victory. To facilitate interest in wagering on such a match, a posted line may appear as

$$
\begin{array}{lll}
\text { Team A } & -l & -110 \\
\text { Team B } & +l & -110 .
\end{array}
$$

The line (1) is based on American odds and stipulates that a wager of $\$ 110$ placed on Team A returns the original $\$ 110$ plus an additional $\$ 100$ if Team A wins by more than $l$ points. Alternatively, a wager of $\$ 110$ placed on Team B returns the original $\$ 110$ plus an additional $\$ 100$ if Team B wins or if Team B loses by less than $l$ points. In the case where Team A wins by exactly $l$ points, the original bets are returned. The quantity $l$ is referred to as the pointspread and is determined by the bookmaker, i.e. the individual or organization that posts the line and collects the bets.

We point out a few variations in the above example. First, different odds may be given. For example, American odds of -120 stipulate that a winning wager of $\$ 120$ returns the original $\$ 120$ plus an additional $\$ 100$. When the American odds are positive, this suggests that an event is less likely. For example, American odds of +140 stipulate that a winning wager of $\$ 100$ returns the original $\$ 100$ plus an additional $\$ 140$. Second, wagers can be made in multiples or fractions of the amounts discussed. Third, a nearly equivalent expression of (1) is based on European odds and appears as

$$
\begin{array}{lll}
\text { Team A } & -l & 1.91 \\
\text { Team B } & +l & 1.91
\end{array} .
$$


Here, a winning wager of $x$ dollars returns $x(1.91)$ dollars. In the case of a $\$ 110$ wager, the return is $\$ 110(1.91)=\$ 210.10$ which is nearly equivalent to the $\$ 110+\$ 100=\$ 210$ situation described above.

Having discussed the betting procedure, it is important to understand the objective of the bookmaker. In (2), suppose that a total of $y$ dollars is wagered on Team A and a total of $y$ dollars is wagered on Team B. In this case, the bookmaker collects $2 y$ dollars, and given that a winner is decided, the bookmaker pays out $y(1.91)$ dollars. The bookmaker has made a profit of $y(0.09)$ dollars regardless of the winning team and the percentage profit (vigorish) is calculated as $y(0.09) /(2 y) \rightarrow 4.5 \%$. Thus, in the case of the line in (2), the 'safe' strategy for the bookmaker is to attempt to select the pointspread $l$ so as to balance the total bets placed on Team A and on Team B for this guarantees a profit. It is this fact that suggests opportunities for the gambler since the bookmaker is not trying to achieve an optimal line from the point of view of prediction. Rather, the bookmaker is trying to assess public opinion (i.e. determine the pointspread $l$ ) so as to balance the bets. A gambler then 'simply' needs to (a) have more insight on reality than the rest of the gambling public and (b) win often enough to overcome the vigorish. Unlike most mechanical games (e.g. roulette), it is evident that the possibility exists to develop winning strategies when gambling on the outcome of sporting events. Stern (1998) suggests that bookmakers are good at setting lines in the sense that actual winning percentages closely resemble the probabilities implied by the lines. For example, he claims that it is reasonable to approximate the outcome of a National Football League game using the normal distribution with mean equal to the point spread and standard deviation equal to 13.5 .

A simple heuristic for wagering is to develop a procedure for constructing pointspreads. When one's personal pointspread differs sufficiently from the pointspread in the posted 
line, this signals a condition to wager. The size of the wager may depend on the magnitude of the departure. We note in passing that the stated heuristic of comparing one's personal pointspread to a bookmaker's pointspread is an application of subjective probability. In fact, it is difficult to imagine how such an application can be reconciled in terms of a frequentist notion of probability.

Finally, we mention two other types of wagers commonly available in the same match involving Team A and Team B. First, odds are usually posted for Team A winning outright (i.e. without a pointspread). Odds are also posted for Team B winning outright. This pair of odds is known as the moneyline. Second, there is usually a total $t$ associated with the match together with under and over odds. An under wager wins if fewer than $t$ total points are scored in the match and an over wager wins if more than $t$ total points are scored in the match. If exactly $t$ total points are scored in the match, the wagers are refunded.

\section{Fixed wagers}

In fixed wagering, without loss of generality, a gambler bets 1 unit on the outcome of each match. Given a gambling system with probability $0<p \leq 1$ of choosing winners and European odds $\theta>1$ in each of the matches, we are interested in the bankroll $B_{m}$ that is realized after placing $m$ bets.

Consider first the simplest situation where the gambler has an initial bankroll $B_{0}$ exceeding $m$ (i.e. the gambler bets within his means and is prepared to lose each of the $m$ bets). In this case, given $m$ independent bets, it is straightforward to write the binomial probability for the bankroll $B_{m}$ as

$$
\operatorname{Pr}\left(B_{m}=B_{0}+j(\theta-1)-(m-j)\right)=\left(\begin{array}{c}
m \\
j
\end{array}\right) p^{j}(1-p)^{m-j} \quad j=0, \ldots, m .
$$


From the binomial distribution, we have $\mathrm{E}\left(B_{m}\right)=B_{0}+m(\theta p-1)$ and $\operatorname{var}\left(B_{m}\right)=\theta^{2} m p(1-$ $p$ ). The normal approximation to the binomial then suggests

$$
B_{m} \stackrel{\mathrm{d}}{=} \mathrm{N}\left(B_{0}+m(\theta p-1), \theta^{2} m p(1-p)\right)
$$

from which probabilities may be calculated. For example, the probability of realizing a profit after $m$ bets is $\operatorname{Pr}\left(B_{m}>B_{0}\right) \approx \Phi\left(m(\theta p-1) / \sqrt{\theta^{2} m p(1-p)}\right)$. We might also use a continuity correction in the calculation of $\operatorname{Pr}\left(B_{m}>B_{0}\right)$.

In practice, a gambler does not know the probability $p$ of choosing winners. Although $p$ may be estimated from historical data, perhaps the most useful applications of (3) and subsequent formulae contained in this paper are sensitivity analyses. A gambler may investigate the range of $p$ necessary to produce worthwhile profits. A gambler may also investigate the size of the loss if the system yields values of $p$ unable to overcome the vigorish. For example, with 'even' odds such as those in (2), the gambler might be willing to assume a worst case scenario of $p=0.5$ for this corresponds to no knowledge whatsoever (i.e. choosing Team A or Team B strictly by the flip of a coin).

We now turn to the more complicated situation where the gambler has an initial bankroll of $B_{0}$, where $0<B_{0}<m$. We make calculations a little simpler by assuming that if after bet $i=1, \ldots, m$, the gambler's bankroll is $0<B_{i}<1$, then the gambler's next bet remains 1 , and the gambler is able to cope with a loss on this bet by borrowing $1-B_{i}$. We say that a gambler is ruined and ceases betting if $B_{i} \leq 0$ for any $i=1, \ldots, m$.

We note that the above setup corresponds to a simple extension of the classical drunkard's walk. In this case, the drunkard, instead of taking steps of size \pm 1 each with probability $\frac{1}{2}$, takes an upward step of size $\theta-1$ with probability $p$ and a downward step of size -1 with probability $1-p$. The probability of ruin within $m$ bets corresponds to the probability that the drunkard hits the absorbing barrier $-B_{0}$ within $m$ steps. For a 
discussion of more general versions of the drunkard's walk, see Feller (1968, sect. 14.8).

For this problem, the distribution of the final bankroll $B_{m}$ is expressable via a recursion. For $i=1, \ldots, m$ and $j=0, \ldots, i$, we define

$$
\begin{aligned}
Q_{j, i-j} & =\operatorname{Pr}\left(B_{i}=B_{0}+j(\theta-1)-(i-j)\right) \\
& =\operatorname{Pr}(j \text { upward steps, }(i-j) \text { downward steps, no ruin }) \\
& = \begin{cases}p Q_{j-1, i-j}+(1-p) Q_{j, i-j-1} & j>\frac{i-B_{0}}{\theta} \\
0 & j \leq \frac{i-B_{0}}{\theta}\end{cases}
\end{aligned}
$$

where $Q_{0,0}=1$ and $Q_{-1, k}=Q_{k,-1}=0$ for all $k$. This forms a tree structure where $Q_{j, i-j}$ is the $j$ th entry along the $i$ th row from the top of the tree. In this case, we construct the tree beginning with the top element $Q_{0,0}$ and work downward along rows where the last row $\left\{Q_{j, m-j}: j=0, \ldots, m\right\}$ gives the probabilities of interest. We note that the probability of ruin is available from the final row via $\operatorname{Pr}($ ruin $)=1-\sum_{j=0}^{m} Q_{j, m-j}$.

Whereas much of the early work corresponding to the drunkard's walk concerned itself with approximate solutions and detailed mathematics, we note that the approach described here is based on ready access to a computing environment; the computational approach is fast and involves straightforward programming. For example, with $\theta=1.91$, $B_{0}=5, p=0.56$ and $m=500$, we obtain $\operatorname{Pr}($ ruin $)=0.428$ and this was obtained nearly instantaneously running Fortran on a SUN workstation.

\section{Fixed percentage wagers}

In simple fixed percentage wagering, a gambler bets a fraction $0 \leq f \leq 1$ of the bankroll on the outcome of a single match. When the outcome of the match is determined, the gambler resumes fixed percentage wagering on the new balance. Assuming the infinite divisibility of money, one of the immediate attractions of fixed percentage wagering is that 
the gambler never goes into debt.

In the context of information theory, Kelly (1956) provided a neat result that is often quoted but misused in gambling circles. Given a gambling system with probability $0<$ $p \leq 1$ of choosing winners and European odds $\theta>1$, Kelly showed that the 'optimal' betting fraction is

$$
f^{*}=\frac{p \theta-1}{\theta-1}
$$

provided that $p>1 / \theta$. For example, consider a system that historically picks winners $54 \%$ of the time (i.e. $p=0.54$ ) with the standard European odds payout of $\theta=1.91$. In this case, the optimal betting fraction is $3.45 \%$ of the bankroll. The Kelly criteria is optimal from several points of view; for example, it maximizes the exponential rate of growth and it provides the minimal expected time to reach a preassigned balance (Breiman, 1961).

Breiman (1961) investigated the properties of betting systems and considered a more general wagering scenario than the simple situation discussed above. However, Breiman's work did not provide the derivation of solutions nor sharp statements concerning the uniqueness of solutions under the general framework.

We are concerned with restricted problems that are of real interest to the sports bettor. For example, it is likely that a sports bettor would like to bet on several matches in a single day. If the bettor uses the Kelly fraction (4) on $n$ such matches where $n f^{*}>1$, then the total amount to bet would exceed the current bankroll. The problem then is to determine fixed percentages that satisfy some optimality.

We therefore consider the situation where on day $j=1, \ldots, m$, the gambler wishes to place $n_{j i}$ wagers on matches with European odds $\theta_{i}$ and where the probability of picking winners is $p_{i}, i=1, \ldots, k$. For example, it is possible that a gambler has a system for betting the pointspread and another system for betting totals (i.e. $k=2$ ). The question 
arises as to what are the optimal betting fractions $f_{j 1}^{*}, \ldots, f_{j k}^{*}$ on day $j$ where $n_{j i}$ wagers are placed with a fraction $f_{j i}$ of the bankroll, $i=1, \ldots k$ ? Given an initial bankroll $B_{0}$, the bankroll at the completion of day $j$ is

$$
B_{j}=\prod_{x_{j 1}=0}^{n_{j 1}} \cdots \prod_{x_{j k}=0}^{n_{j k}}\left(\left(1-\sum_{i=1}^{k} n_{j i} f_{j i}\right) B_{j-1}+\sum_{i=1}^{k} x_{j i} \theta_{i} f_{j i} B_{j-1}\right)^{\Delta_{j i}}
$$

where $\Delta_{j i}=I\left(X_{j i}=x_{j i}\right), i=1, \ldots, k$, in which the random variable $X_{j i}$ denotes the number of winning wagers of type $i$ on day $j$. Therefore, only one term in the product (5) is not equal to 1 . Note also that the first term in the outer parentheses is the balance of the bankroll not bet in a given day and we require $\sum_{i=1}^{k} n_{j i} f_{j i}<1$ to prevent the possibility of bankruptcy.

Assuming that $X_{j 1}, \ldots, X_{j k}$ are independent with $X_{j i} \sim \operatorname{Bi}\left(n_{j i}, p_{i}\right), i=1, \ldots, k$, we are concerned with the maximization of the function $G=G\left(f_{j 1}, \ldots, f_{j k}\right)=\mathrm{E}\left(\log \left(B_{j} / B_{j-1}\right)\right)$ where $G$ is referred to as the exponential rate of growth (Breiman, 1961) and

$$
\begin{aligned}
G & =\mathrm{E}\left(\sum_{x_{j 1}=0}^{n_{j 1}} \cdots \sum_{x_{j k}=0}^{n_{j k}} \Delta_{j i} \log \left(1-\sum_{i=1}^{k} n_{j i} f_{j i}+\sum_{i=1}^{k} x_{j i} \theta_{i} f_{j i}\right)\right) \\
& =\sum_{x_{j 1}=0}^{n_{j 1}} \cdots \sum_{x_{j k}=0}^{n_{j k}}\left(\prod_{i=1}^{k}\left(\begin{array}{l}
n_{j i} \\
x_{j i}
\end{array}\right) p_{i}^{x_{j i}}\left(1-p_{i}\right)^{n_{j i}-x_{j i}}\right) \log \left(1+\sum_{i=1}^{k} f_{j i}\left(x_{j i} \theta_{i}-n_{j i}\right)\right) .
\end{aligned}
$$

In the Appendix, we establish the existence of a unique maximum $\mathbf{f}^{*}=\left(f_{j 1}^{*}, \ldots, f_{j k}^{*}\right)$ and provide a simple algorithm that evaluates $\mathbf{f}^{*}$. For example, consider a betting system where $k=3, n_{j 1}=2, n_{j 2}=3, n_{j 3}=3, p_{1}=0.545, p_{2}=0.565, p_{3}=0.585$ and $\theta_{1}=\theta_{2}=\theta_{3}=1.91$. The algorithm gives $f_{j 1}^{*}=0.0416, f_{j 2}^{*}=0.0811$ and $f_{j 3}^{*}=0.1215$. The proposed algorithm has worked well in all of the examples that we have considered. We note however that convergence difficulties were experienced with more sophisticated 
algorithms taken from numerical libraries. This is due to the fact that in many practical situations, the function $G$ is nearly flat in neighbourhoods of $\mathbf{f}^{*}$. As a result, successive values $\mathbf{f}^{(1)}, \mathbf{f}^{(2)}, \ldots$ based on derivatives or finite difference methods may oscillate around the maximum. For example, the IMSL routine duminf based on a quasi-Newton method failed to converge for the problem described above. The lesson, as always, is that it is sensible to use available information (e.g. the shape of $G$ ) in optimization problems.

Now that a gambler is equiped with an algorithm for determining optimal fixed percentages, the gambler may want to investigate the future performance of the betting strategy used over a season. In other words, it would be useful to have some distributional results concerning the final bankroll. Referring to (5), we can express the final bankroll as

$$
\begin{aligned}
B_{m} & =\left(1-\sum_{i=1}^{k} n_{m i} f_{m i}\right) B_{m-1}+\sum_{i=1}^{k} X_{m i} \theta_{i} f_{m i} B_{m-1} \\
& =B_{0} \prod_{j=1}^{m}\left(1+\sum_{i=1}^{k} f_{j i}\left(X_{j i} \theta_{i}-n_{j i}\right)\right) .
\end{aligned}
$$

In assessing a system before the season begins, expression (7) is useful for simulation purposes. Of course, the bettor does not know in advance the number $n_{j i}$ of wagers of type $i$ on a given day $j$, and therefore, some distribution on $n_{j i}$ is assigned. The simulation then proceeds by generating $n_{j i}$ and then generating $X_{j i} \stackrel{\mathrm{d}}{=} \operatorname{Bi}\left(n_{j i}, p_{i}\right), j=1, \ldots, m$, $i=1, \ldots, k$. Using $(7)$, this determines a single variate $B_{m}$. The procedure is repeated to build up the distribution of $B_{m}$.

It might be assumed that under repeated wagering, a large sample result may hold and that the distribution of the final bankroll $B_{m}$ in $(7)$ is approximately normal. To see that this may be far from the truth, consider the following simple situation where $m=162$, $k=2, p_{i}=0.56, \theta_{i}=1.91$ and $n_{j i}=1$ for all $i, j$. This corresponds to a successful betting 
system for the 2000/2001 National Basketball Association season where the bettor places a single wager on each of two types of bets every day of the season. The optimal Kelly fraction (4) is $f_{j i}^{*}=0.0761$. Using the simulation procedure based on 1000 simulations, Figure 1 provides a histogram of the final bankroll $B_{162}$ using an initial bankroll $B_{0}=100$. Figure 1 suggests that at the end of the season, the proposed system will probably yield a small profit (i.e. a final bankroll of less than 1000) although the possibility does exist for huge profits. We also observe that the final bankroll has a distinctly non-normal distribution with a very long right tail. However, it is clear that $\log B_{m}$ is a sum of random variables. Therefore the Central Limit Theorem suggests that $\log B_{m}$ may be approximately normal. Using the same example as above, Figure 2 provides a histogram of $\log B_{162}$. The histogram appears more normal and the $\log$ data passes the AndersonDarling goodness-of-fit test for normality yielding a $P$-value exceeding 0.5 .

We can evaluate the mean $\mathrm{E}\left(B_{m}\right)$ using the formula involving conditional expectations:

$$
\mathrm{E}\left(B_{m}\right)=B_{0}\left(1+\sum_{i=1}^{k} \mathrm{E}\left(n_{j i} f_{j i}\right)\left(p_{i} \theta_{i}-1\right)\right)^{m}
$$

where it is assumed that the expectation (8) does not depend on the day $j$. 


\section{Is a gambling system profitable?}

Our focus now turns to another practical question: is a proposed gambling system profitable? Typically, a thoughtful sports bettor, would like to test a gambling system using historical data before risking his own money. Mok (2001) considers this problem in more detail than presented here. Consider then a match with European odds

\section{Team A $\theta_{A}$ \\ Team B $\theta_{B}$}

where without loss of generality, we ignore the pointspread. If $p_{A}$ is the true probability that Team A wins and $p_{B}=1-p_{A}$ is the true probability that Team B wins (ignoring ties), then we should bet on Team A if the expected profit from betting on Team A is positive. In other words, we should bet on Team A if

$$
\left(\theta_{A}-1\right) p_{A}+(-1)\left(1-p_{A}\right)>0 \quad \rightarrow \quad p_{A}>1 / \theta_{A} .
$$

Similarly, we should bet on Team B if $p_{B}>1 / \theta_{B}$. Recall that $p_{A}$ and $p_{B}$ are unknown and the inequality $1 / \theta_{A}+1 / \theta_{B}>1$ is due to the vigorish.

Now we first test the profitability of a gambling system in a restricted context. Suppose that we have the results of $n$ historical matches where the proposed gambling system would have bet on Team A with European odds as in (9). Suppose further that $x$ of the $n$ bets are winning bets. Then we test for a profitable system by considering $H_{0}: p_{A} \leq 1 / \theta_{A}$ versus $H_{1}: p_{A}>1 / \theta_{A}$. The corresponding $P$-value is given by the binomial probability

$$
\operatorname{Pr}(X \geq x)=\sum_{i=x}^{n}\left(\begin{array}{c}
n \\
i
\end{array}\right)\left(1 / \theta_{A}\right)^{i}\left(1-1 / \theta_{A}\right)^{n-i}
$$


where a small $P$-value indicates evidence of a profitable system. This is a simple procedure to determine whether historical data provides evidence of long-term profitability. For example, if there are $x=60$ winning wagers out of $n=100$ matches with standard payout $\theta_{A}=1.91$, then $p_{A}=1 / \theta_{A}=0.524$ and we have mild evidence $(P=0.076)$ of a profitable system. Remarkably, tests such as these are rarely (perhaps never) discussed in the myriad of gambling books available at the Gamblers Book Shop (www.gamblersbook.com), a key source for information on gambling.

The situation becomes more complex when bets are placed at various odds. We have collected data arising from the 2000 Major League Baseball season for which a proposed betting system was established. Ignoring some of the details, personal odds were constructed corresponding to Team A defeating Team B. These odds were based on logistic regression using the pitchers' earned run averages, overall team batting averages and team winning percentages as covariates. When the personal odds differed from the posted odds at the Intertops website (www.intertops.com) by more than $10 \%$, this signaled a condition to wager.

Table 1 gives the data and $P$-values for groups of bets placed at 24 differents sets of odds. The $P$-values are obtained using (10). We observe that only one of the results are significant. The problem now is to determine whether the overall system is profitable. A standard approach is based on Fisher's test (D'Agostino \& Stephens, 1986 p. 357). Following the notation above, let $H_{0 k}\left(H_{1 k}\right)$ denote the hypothesis that the $k$ th type of bet is unprofitable (profitable) and let $q_{k}$ denote the corresponding $P$-value, $k=1, \ldots, 24$. Fisher's test considers the overall null hypothesis $\Omega_{0}=\bigcap_{k=1}^{24} H_{0 k}$ versus the alternative hypothesis that at least one of the $H_{0 k}$ is false. Applying Fisher's test to the data in Table 1, we obtain the overall $P$-value $\operatorname{Pr}\left(\chi_{48}^{2}>-2 \sum_{k=1}^{24} \log q_{k}\right)=\operatorname{Pr}\left(\chi_{48}^{2}>52.767\right)=0.295$ which does not allow us to reject the overall null hypothesis $\Omega_{0}$ that all of the component 
types of bets are unprofitable.

The approach above is not quite right. With respect to the data in Table 1, there is not enough evidence to reject $\Omega_{0}$, and even if there was, this does not imply that the gambling system is profitable. We define a profitable gambling system as one for which all of the $H_{1 k}$ are true. Therefore we should instead test $\Omega_{0}$ versus the alternative $\Omega_{1}=\bigcap_{k=1}^{24} H_{1 k}$ and exclude regions of the parameter space that do not belong in $\Omega_{0} \cup \Omega_{1}$. We exclude such regions because the gambling system uses the same betting criteria regardless of the odds and it is therefore logical that if it is an unprofitable (profitable) gambling system, then it should be an unprofitable (profitable) system for all component types of bets. We emphasize that the relevant problem is to test a hypothesis $\Omega_{0}$ which is a small set where all component bets are unprofitable versus a hypothesis $\Omega_{1}$ which is a small set where all component bets are profitable.

To test $\Omega_{0}$ versus $\Omega_{1}$ in a classical framework, it is natural to reject $\Omega_{0}$ based on large values of the generalized likelihood ratio statistic

$$
\Lambda=\frac{\sup _{\Omega_{0} \cup \Omega_{1}} L(x)}{\sup _{\Omega_{0}} L(x)}
$$

where $L(x)$ is the corresponding product probability mass function based on the data $x$. The exact discrete distribution of $\Lambda$ is beyond reach since the sample space is so large. Also, we cannot appeal to a large sample $\chi^{2}$ distribution for $2 \log \Lambda$ since $\operatorname{dim}\left(\Omega_{0}\right)=$ $\operatorname{dim}\left(\Omega_{1}\right)$ which means that we have 'zero' degrees of freedom. In addition, the supremums in $\Lambda$ occur on the boundaries of the parameter space and this invalidates necessary large sample assumptions.

We consider now a Bayesian approach to testing $\Omega_{0}$ versus $\Omega_{1}$. The Bayesian model contains a little more structure due to the necessity of prior distributions. Given the European odds (9), we assume that over many matches the odds are set such that bets 
Table 1: Betting results using a proposed gambling system; $\theta_{A}$ is the posted odds for Team $\mathrm{A}, \theta_{B}$ is the posted odds for Team B, $x$ is the number of wins by betting on Team $\mathrm{A}$ and $n$ is the total number of corresponding matches. The $P$-value is given and an asterisk indicates significance at level 0.05 .

\begin{tabular}{rrrrl}
\hline$\theta_{A}$ & $\theta_{B}$ & $x$ & $n$ & $P$-value \\
& & & & \\
\hline & & & & \\
2.800 & 1.455 & 12 & 32 & 0.482 \\
2.700 & 1.498 & 11 & 25 & 0.299 \\
2.650 & 1.541 & 10 & 23 & 0.357 \\
2.600 & 1.556 & 8 & 18 & 0.384 \\
2.550 & 1.571 & 13 & 34 & 0.610 \\
2.500 & 1.588 & 17 & 35 & 0.193 \\
2.450 & 1.606 & 13 & 38 & 0.840 \\
2.400 & 1.625 & 44 & 89 & 0.084 \\
2.350 & 1.645 & 19 & 43 & 0.472 \\
2.300 & 1.666 & 26 & 56 & 0.376 \\
2.250 & 1.690 & 28 & 59 & 0.367 \\
2.200 & 1.714 & 39 & 65 & $0.013^{*}$ \\
2.150 & 1.740 & 22 & 43 & 0.322 \\
2.100 & 1.770 & 23 & 41 & 0.176 \\
2.050 & 1.800 & 26 & 47 & 0.226 \\
2.000 & 1.833 & 20 & 39 & 0.500 \\
1.910 & 1.910 & 9 & 17 & 0.578 \\
1.833 & 2.000 & 12 & 20 & 0.399 \\
1.800 & 2.050 & 4 & 7 & 0.620 \\
1.770 & 2.100 & 29 & 51 & 0.538 \\
1.740 & 2.150 & 4 & 6 & 0.492 \\
1.690 & 2.250 & 3 & 3 & 0.207 \\
1.645 & 2.350 & 3 & 5 & 0.696 \\
1.625 & 2.400 & 3 & 5 & 0.709 \\
\hline & & & &
\end{tabular}


on Team A and Team B are equally attractive (i.e. they yield the same expected return). Therefore, based on a unit bet,

$$
\left(\theta_{A}-1\right) p_{A}+(-1)\left(1-p_{A}\right)=\left(\theta_{B}-1\right)\left(1-p_{A}\right)+(-1) p_{A} \quad \rightarrow \quad p_{A}=\theta_{B} /\left(\theta_{A}+\theta_{B}\right)
$$

where $\theta_{B} /\left(\theta_{A}+\theta_{B}\right)$ represents the expected probability that Team A wins and the expectation is taken over many matches. In any particular match, the true probability $p_{A}$ may be something different than $\theta_{B} /\left(\theta_{A}+\theta_{B}\right)$. Let $X_{k}$ denote the number of winning bets of type $k$ out of $n_{k}$ wagers and let $p_{k}^{(0)}=\theta_{B} /\left(\theta_{A}+\theta_{B}\right)$ for the $k$ th type of bet, $k=1, \ldots, N$. For the data in Table $1, N=24$. This suggests the hierarchical model

$$
\begin{array}{rll}
\left(X_{k} \mid p_{k}\right) & \stackrel{\mathrm{d}}{=} \operatorname{Bi}\left(n_{k}, p_{k}\right) & k=1, \ldots, N \\
\left(p_{k} \mid m\right) & \stackrel{\mathrm{d}}{=} \beta\left(m p_{k}^{(0)}, m\left(1-p_{k}^{(0)}\right)\right) & k=1, \ldots, N \\
m & \stackrel{\mathrm{d}}{=} \mathrm{U}(l, \infty) & \text { where } l=\max _{k}\left(1 / p_{k}^{(0)}, 1 /\left(1-p_{k}^{(0)}\right)\right)
\end{array}
$$

where the $p_{k}$ are conditionally independent. The beta prior for $p_{k}$ is reasonable from the point of view that $p_{k}$ is constrained to the interval $(0,1)$ and $\mathrm{E}\left(p_{k} \mid m\right)=p_{k}^{(0)}$ as argued previously. The hyperparameter $m$ controls the variance of the $p_{k}$, and we assign a flat improper prior for $m$. We include the lower limit on $m$ as this forces concave densities for $p_{1}, \ldots, p_{N}$.

The hierarchical model (11) induces a $(N+1)$ dimensional posterior distribution given by $(\mathbf{p}, m \mid \mathbf{x})$. Inference based on $(\mathbf{p}, m \mid \mathbf{x})$ is straightforward using the Gibbs sampling algorithm as the full conditional distributions of the $p_{k}$ are beta distributions and $m$ can be generated in closed form from its full conditional distribution via inversion. To obtain the posterior probabilities of $\Omega_{0}$ and $\Omega_{1}$, we simply calculate the proportion of the generated ps that fall into the two respective sets. For the data in Table 1, it turns out 
that both of these probabilities are essentially zero.

We are therefore faced with the problem of assessing two hypotheses $\Omega_{0}$ and $\Omega_{1}$, one of which we believe to be true, when both hypotheses correspond to very improbable sets. This is a general problem of inference which goes beyond the application considered here. Our approach which borrows on ideas from Swartz (1999) and Evans et al. (1997) is to generate $\mathbf{p}$ from the Gibbs sampling algorithm and then calculate its Euclidean distance $D_{0}$ from $\Omega_{0}$ and its Euclidean distance $D_{1}$ from $\Omega_{1}$. The smaller (larger) the quantity $D_{0} / D_{1}$, the more evidence $\mathbf{p}$ provides in favour of the hypothesis $\Omega_{0}\left(\Omega_{1}\right)$. We therefore consider posterior probabilities $\operatorname{Pr}\left(D_{0} / D_{1} \leq t \mid \mathbf{x}\right)$ for different values of $t$. To put more reliance on the data we also calculate the Bayes factor

$$
\mathrm{BF}_{D}=\frac{\operatorname{Pr}\left(D_{0} / D_{1} \leq t \mid \mathbf{x}\right)}{\operatorname{Pr}\left(D_{0} / D_{1}>t \mid \mathbf{x}\right)} \frac{\operatorname{Pr}\left(D_{0} / D_{1}>t\right)}{\operatorname{Pr}\left(D_{0} / D_{1} \leq t\right)}
$$

where small (large) values give evidence in favour of $\Omega_{1}\left(\Omega_{0}\right)$. There is one difficulty with the calculation of $\mathrm{BF}_{D}$ and this concerns the improper prior for $m$. With an improper prior, we are unable to generate from the joint prior distribution of $(\mathbf{p}, m)$ to obtain $\operatorname{Pr}\left(D_{0} / D_{1} \leq t\right)$ and $\operatorname{Pr}\left(D_{0} / D_{1}>t\right)$. For the data in Table 1 , we therefore set $m=2.98$ which is the posterior mean of $m$ estimated from the output of the Gibbs sampling algorithm, and we then simulate the $p_{k} \mathrm{~s}$ from the beta priors with this value of $m$. Table 2 shows $\mathrm{BF}_{D}$, the posterior odds of $D_{0} / D_{1} \leq t$ and the prior odds of $D_{0} / D_{1} \leq t$ corresponding to the data in Table 1. As expected, we see that both the posterior odds and prior odds are increasing in $t$. However, the prior odds are larger and increase more rapidly than the posterior odds. This causes the Bayes factor $\mathrm{BF}_{D}$ to be small and approach zero as $t$ increases. We also observe that $\mathrm{BF}_{D}$ has its largest value when $t$ is around 1 .

Now if you have confidence that the prior is realistic, then it is often argued that inference should be based solely on the posterior as Bayes Theorem provides the recipe 
Table 2: $\mathrm{BF}_{D}$, the posterior odds of $D_{0} / D_{1} \leq t$, the prior odds of $D_{0} / D_{1} \leq t$ for selected values of $t$ using the data in Table 1 .

\begin{tabular}{rrrr}
\hline & & & \\
& & & \\
& & & \\
0.6 & 0.009 & 0.001 & 0.114 \\
0.7 & 0.030 & 0.008 & 0.271 \\
0.8 & 0.035 & 0.018 & 0.522 \\
0.9 & 0.046 & 0.040 & 0.859 \\
1.0 & 0.051 & 0.081 & 1.591 \\
1.2 & 0.048 & 0.203 & 4.208 \\
1.4 & 0.036 & 0.410 & 11.500 \\
1.6 & 0.025 & 0.631 & 25.316 \\
1.8 & 0.014 & 0.996 & 70.429 \\
2.0 & 0.012 & 1.532 & 124.000 \\
2.5 & 0.006 & 3.219 & 499.000 \\
3.0 & 0.000 & 5.410 & $\infty$ \\
4.0 & 0.000 & 13.925 & $\infty$ \\
6.0 & 0.000 & 70.429 & $\infty$ \\
8.0 & 0.000 & 165.667 & $\infty$ \\
\hline
\end{tabular}


for combining information from the data (i.e. the likelihood) and information from the prior. In this case, the posterior odds from Table 2 clearly suggest that the gambling system is profitable based on the data in Table 1. Note that using the posterior odds for $t=1.0$ in Table 2 , we obtain $\operatorname{Pr}\left(D_{0}<D_{1} \mid \mathbf{x}\right)=0.075$.

Now if you have less faith in your prior, then you may want to base your inference on the Bayes factor as it relies more strongly on the data by factoring out the prior odds. Since the Bayes factor $\mathrm{BF}_{D}$ is very small for different values of $t$ in Table 2, this gives strong evidence in favour of $\Omega_{1}$ under the assumption that $\Omega_{0}$ and $\Omega_{1}$ are the only

possibilities in the parameter space. For example, observe that the Bayes factor is roughly at least 20 times more favourably inclined toward $\Omega_{1}$ than $\Omega_{0}$. Therefore, in contrast to the previous analyses, the Bayesian analyses using both posterior probabilities and Bayes factors suggest that the data in Table 1 provide evidence of a profitable system.

The underlying practical problem has lead to a significant theoretical problem involving the testing of non-standard hypotheses.

\section{Discussion}

Now suppose that you have a 'winning' system. How should you bet? Typically, Sportsbooks assign an upper limit on wagering. Since the Kelly approach has an optimal rate of growth, it seems logical to begin with the Kelly system using fixed percentage wagering until the upper limit is attained. As long as the Kelly system prescribes a bet exceeding the upper limit, use fixed wagering with the upper limit.

Sportsbooks also typically assign a lower limit on wagering. For some internet sites, the lower limit is so low (e.g. $\$ 1$ at www.intertops.com) that it can be practically ignored. We recommend an alternative betting strategy that also allows us to ignore the lower limit on wagering. Suppose that you have an initial bankroll $B_{0}=\$ 500$ and that you are 
wagering at a Sportsbook with a lower limit of $\$ 10$ and an upper limit of $\$ 3000$. Extensive personal simulations have shown that it is better to begin with the Kelly system using a bankroll of $x_{1}=\$ 400$, and if the bankroll drops below $x_{2}=\$ 200$, add the final $\$ 100$ to the bankroll. The idea is to 'kickstart' the system since a very small bankroll grows slowly with fixed percentage wagering. It would be interesting to see if optimal values for $x_{1}$ and $x_{2}$ could be obtained. Again, as long as the prescribed value of Kelly wagering exceeds $\$ 3000$, you would maintain $\$ 3000$ betting.

The results that we have presented in this paper are readily applicable to sports betting. The real difficulty is coming up with a winning system (i.e. a system where $p$ is sufficiently large to overcome the vigorish). Naturally $p$ is unknown, and therefore we might estimate $p$ for a proposed system using past data. Of course, there is no guarantee that results will replicate from year to year, and we need also be wary of multiple comparisons issues when considering various systems.

\section{Appendix: the maximization of $G$}

In the context of simultaneous fixed percentage wagers, we consider the maximization of the function $G$ in (6). The first and second derivatives of $G$ are given by

$$
G_{i}=\frac{\partial G}{\partial f_{j i}}=\sum_{x_{j 1}=0}^{n_{j 1}} \cdots \sum_{x_{j k}=0}^{n_{j k}}\left(\prod_{i=1}^{k}\left(\begin{array}{l}
n_{j i} \\
x_{j i}
\end{array}\right) p_{i}^{x_{j i}}\left(1-p_{i}\right)^{n_{j i}-x_{j i}}\right) \frac{x_{j i} \theta_{i}-n_{j i}}{1+\sum_{i=1}^{k} f_{j i}\left(x_{j i} \theta_{i}-n_{j i}\right)}
$$

and

$G_{i_{1} i_{2}}=\frac{\partial^{2} G}{\partial f_{j i_{1}} \partial f_{j i_{2}}}=\sum_{x_{j 1}=0}^{n_{j 1}} \cdots \sum_{x_{j k}=0}^{n_{j k}}\left(\prod_{i=1}^{k}\left(\begin{array}{c}n_{j i} \\ x_{j i}\end{array}\right) p_{i}^{x_{j i}}\left(1-p_{i}\right)^{n_{j i}-x_{j i}}\right) \frac{-\left(x_{j i_{1}} \theta_{i_{1}}-n_{j i_{1}}\right)\left(x_{j i_{2}} \theta_{i_{2}}-n_{j i_{2}}\right)}{\left(1+\sum_{i=1}^{k} f_{j i}\left(x_{j i} \theta_{i}-n_{j i}\right)\right)^{2}}$

for $i, i_{1}, i_{2}=1, \ldots, k$. Now $G$ is a continuous function defined on the intersection of $[0,1)^{k}$ 
and the halfspace $\sum_{i=1}^{k} n_{j i} f_{j i}<1$. When $p_{i}>1 / \theta_{i}, i=1, \ldots, k$, we obtain

$$
\begin{aligned}
& \text { (a) } G_{i}>0 \text { when } f_{j i}=0, i=1, \ldots, k, \\
& \text { (b) } G_{i_{1} i_{2}}<0 \text { everywhere for } i_{1}, i_{2}=1, \ldots, k \\
& \text { and } \quad \text { (c) } G \rightarrow-\infty \text { as } \sum_{i=1}^{k} n_{j i} f_{j i} \rightarrow 1 .
\end{aligned}
$$

Using these results, and by considering closed sets that approach the region of interest, it can be shown that there is a single critical point lying in the interior of the region, and this point is a global maximum. This establishes the uniqueness of $f_{j 1}^{*}, \ldots, f_{j k}^{*}$. Since $G(0)=0$, we also have that $G\left(\mathbf{f}^{*}\right)>0$.

The shape and smoothness of $G$ yields a simple algorithm which is guaranteed to find $f_{j 1}^{*}, \ldots, f_{j k}^{*}$. We begin by initializing an interior point $f_{j i}=1 / \sum_{i=1}^{k} 2 n_{j i}, i=1, \ldots, k$. To obtain the root of $G_{1}$ along the first coordinate direction, bisection is carried out using the lower starting value $f_{j 1}^{(l)}=0$ and the upper starting value $f_{j 1}^{(u)}$ which intersects the plane $\sum_{i=1}^{k} n_{j i} f_{j i}=1$. After the first coordinate is updated, the procedure is repeated along the coordinate directions $2, \ldots, k$. The loop in this sequential procedure is repeated until the movement in the point is sufficiently small. The maximum has then been obtained. The proposed algorithm is a special case of Gauss-Seidel iteration (Thisted 1988, page 187) where success is based on the recognition that every step of bisection results in a move up the hill and that there are no saddle points or minima of the function $G$.

\section{References}

Breiman, L. (1961). Optimal gambling systems for favorable games. Proceedings of the Fourth Berkeley Symposium on Mathematical Statistics and Probability, Jerzy Neyman, editor, 65-78.

Crist, S. (1998). All bets are off. Sports Illustrated, 88 (3), 82-92. 
D’Agostino, R.B. \& Stephens, M.A. (1986). Goodness-of-Fit Techniques, Marcel Dekker, New York.

Evans, M., Gilula, Z., Guttman, I. \& Swartz, T.B. (1997). Bayesian analysis of stochastically ordered distributions of categorical variables. Journal of the American Statistical Association, 92, 208-214.

Feller, W. (1968). An Introduction to Probability Theory and its Applications, Volume 1, Third Edition, John Wiley and Sons, Inc.

Haywood, H. (2000). BeatWebCasinos.Com: The Shrewd Player's Guide to Internet Gambling, RGE Publishing, Oakland, California.

Kelly, J.L. (1956). A new interpretation of information rate. Bell System Technical Journal, 35, 917-926.

McCune, B. (1989). Education of a Sports Bettor, McCune Sports Investments, Las Vegas, Nevada.

Mok, L. (2001). Testing whether a gambling system is profitable. MSc project, Simon Fraser University, Department of Statistics and Actuarial Science.

Ordine, B. (2000). Super bowl Sunday means lots of action in Las Vegas. Seattle Times, January 23.

Productivity Commission (1999). Australia's gambling industries (Report No 10), PC Inquiry Report, December, www.pc.gov.au/inquiry/gambling/finalreport/index.html.

Stern, H.S. (1998). How accurate are the posted odds?. In the column A Statistician Reads the Sports Pages, Chance, 10 (4), 17-21.

Swartz, T.B. (1999). Nonparametric goodness-of-fit. Communications in Statistics: Theory and Methods, 28, 2821-2841. 
Thisted, R.A. (1988). Elements of Statistical Computing: Numerical Computation, Chapman and Hall, New York.

Thorp, E.O. (1969). Optimal gambling systems for favorable games. Review of the International Statistical Institute, 37, 273-293. 
Figure 1: Histogram of the final bankroll based on 1000 simulations

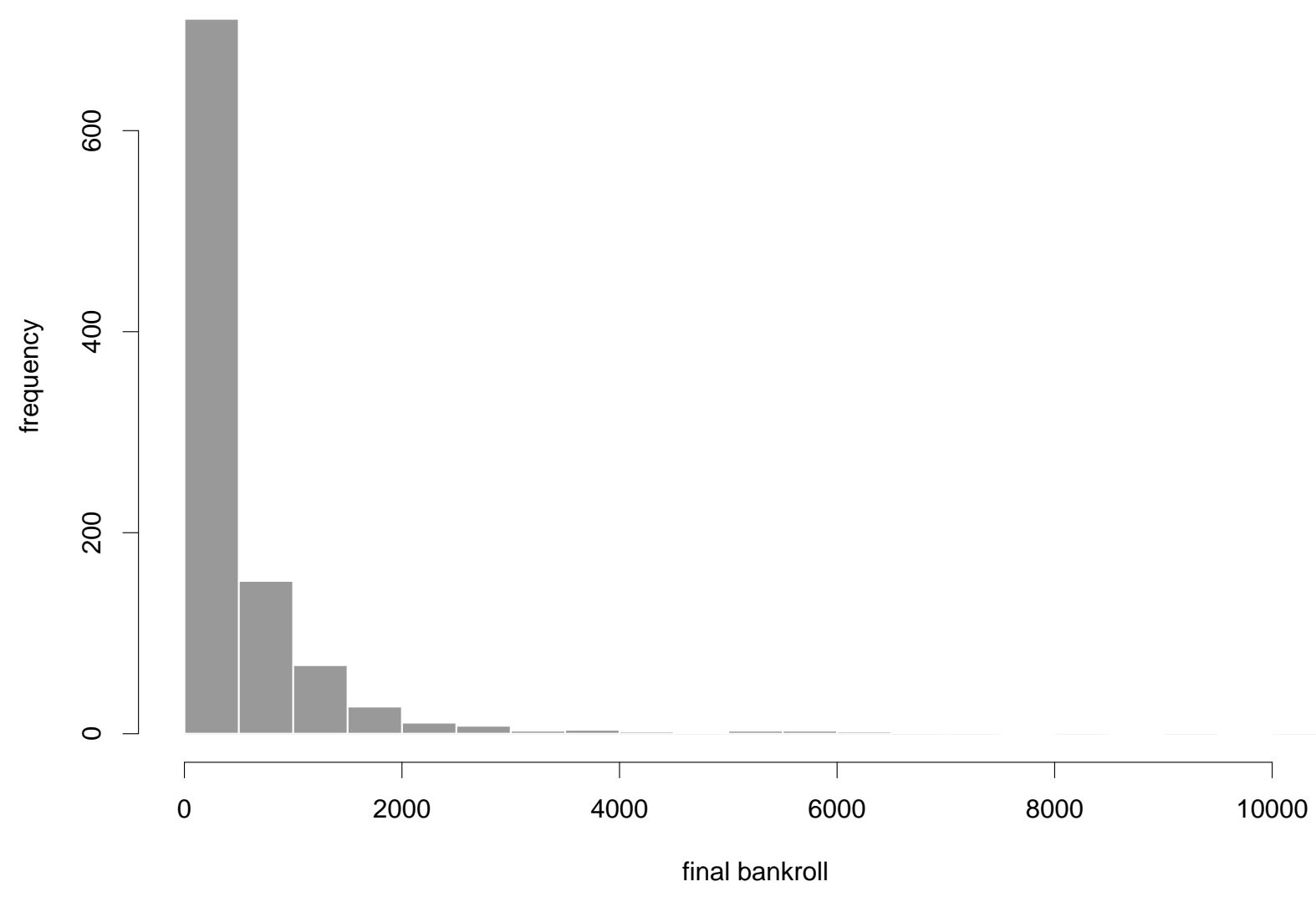


Figure 2: Histogram of the logarithm of the final bankroll based on 1000 simulations

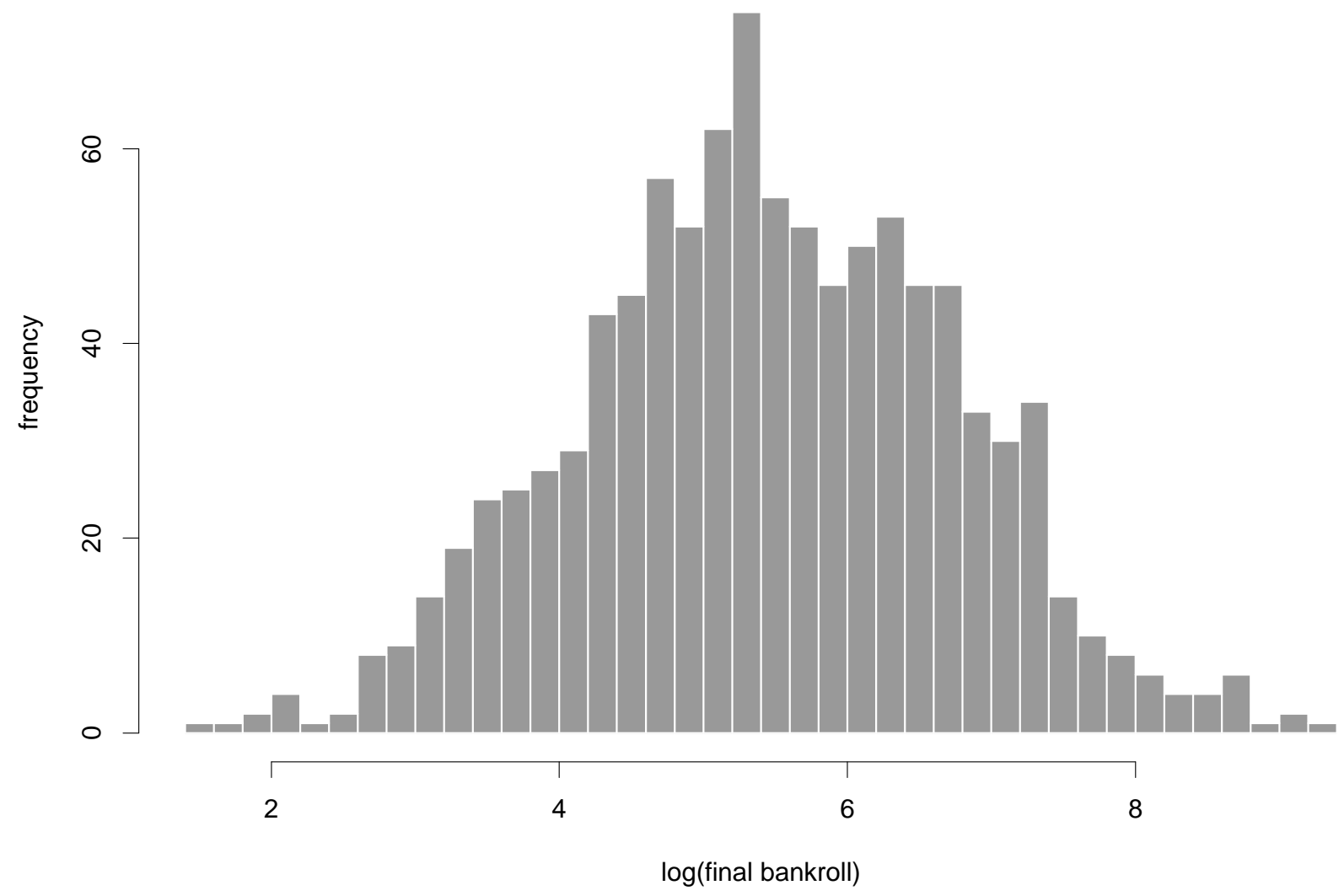

